\title{
VISUALIZATIONS FOR NATURAL SCIENCE EDUCATION
}

Dear Readers!

Motivation, to be successful, and Natural Science Education is no exception in this context should have to rely on a rich repertoire of experiences on which to build conceptual learning by students. These experiences could pave the way to making meaning of them, which in turn supports learning. It also helps to entertain, create or re-create a sense of wonder, which becomes an authentic incentive for learning.

It is therefore important to think of education in a systemic conception, not just limiting the student's experiences to what can possibly take place in the classroom. Therefore, the role of alternative learning environments becomes critical as a prelude, a complement to follow-up the school-based learning process. Experience comes from interaction with a learning environment.

There is great development of hardware that allows us to use powerful computers that support the design of several visualizations with scientific models, especially dynamic models, simulations and interactive modeling software that can be displayed and manipulated in a virtual format and by remote access.

The accelerated development of Information and Communication Technologies in Natural Science Education followed by several studies in the cognitive theory area, have promoted the construction of many visual tools (animations, simulations, games, statics or dynamics virtual 2D and 3D images, multimedia software's, etc.) that have been placed to the disposition of educators.

Nowadays, we can consider the visualization aids and high performance computing have changed the nature of science research and have the promise to transform natural science instruction. As the amount of information acquired through visual tool multiplies, the ability to understand, evaluate, and produce visual representations have become increasingly important in science education.

There are some researches that encourage inserting the computer into the classroom, and they have pointed out that it's necessary because it is modern and there is no way back. Governments around the world have a great program for digital inclusion, focused on the equipment. It seems that when all these projects are implemented we will not have any problems in teaching science at school.

What is more relevant: the computer or the teachers? Of course, when some of these projects don't have success, the problem is the teacher, because they are "old fashioned", they do not know how to use these "fantastic" tools to engage students into the activities.

It does not mean that we have just to take computers into science classrooms, but what kind 
of Natural Science Teacher Education Program we have to prepare them for this contemporary context?

What is it that makes us lower our standards when visual media are concerned while, at the same time, we face the highest demands on texts? Teachers are still "geared" to the written word. They should remember that they face a young generation growing up with images, a generation learning to use computers much more quickly than their parents.

The very first moment when the students are exposed to these visual tools, the visual appeal is too strong. Draw the attention, so the motivation is assured. However, how to move beyond motivation, science lessons need to go beyond motivation, the engagement, they need to provide opportunities for students to build their models, their explanations, interpretations therefore, they must have the chance to express themselves, to communicate their own ideas about the scientific contents.

The use of visualizations in Natural Science Education became a new field of research. In the last years, four very different groups of people (computer software specialists, scientists, educationalists and cognitive scientists) have promoted the development, the discussion and the use of visual tools in natural sciences instruction; nevertheless, research evidences are only in the beginning.

For students in the macroscopic world immersed the reality. It becomes very difficult for them to understand the concepts and the processes located at the submicroscopic level and represented in the symbolic language of natural science. There is a steady growing body of research that suggests that the use of visual tools helps students' transition between these levels of representation, and their achievement in natural science is generally supported by direct access to multimedia modes of representation.

Considering now the use in general of visualizations in science teaching (not only the ones that are supported by ICT), there is an increasing field of research that have been discussing the use of these tools, regarding their importance and influence.

In the teaching of physics, he touches the equipment and appliances. In biology education they can see and touch the bodies, living beings, but what about the teaching of chemistry? How could he see the structural arrangements? The need for recourse to models becomes even more important in science education.

This is a complex issue by itself that often begins with the polysemous definition of the term visualization. There are three common academic usages of visualization in psychology and educational research: external visualization, internal visualization and visualization as a type of spatial skill. In a simple way one can say that external visualizations in science refer to graphics, diagrams, models, simulations, etc., representations typically used in learning; internal visualizations are used to describe internal mental constructs, i.e., mental models and visualization are also used to describe a type of spatial skill. Visualization is seen like the ability to manipulate or transform the image or spatial patterns into other arrangements.

The science teacher needs to play a key role in the negotiation of meaning within educational situations where the visual aids are involved in, when students are making use of models in order to communicate their explanations and show their understanding of science contents.

Teaching and learning toward these tools is a complex process, where teachers need to have some knowledge to use visual tools in a profitable way. As it was mentioned before the situation is becoming more complex regarding the frequent incorporation of the visualization in multimedia environments.

We believe that we were able to soften the naïve vision that these resources can only make classes more pleasant, and so we think that the training had a positive impact on their conceptions about visualizations and we could observe a changing on the reasons and criterion to use these tools and we hope to have contributed to make them more reflexive when using visualizations.

But, considering the insertion of these tools into the natural science classes, it leads us to think: How is a visual representation turned into knowledge? What are the mental processes that 
are involved in attaching a meaning to a representation? What mental and brain processes are involved in "seeing"? Are there different modes of thinking for chemistry, biology, physics, geosciences and etc.?

There are more questions than answers. The role of models and visualization in science and natural science education has been discussed in the last years in several studies: What to study?, How to do so?, What constitutes good practices?, How to evaluate the outcomes of that practice? How to prepare natural science teacher for this context? Are there some of the challenges that researchers have faced and will continue to face in the following years. 